\title{
F wie Fernweh oder f(v)erwöhnt
}

Dominik Heim

Korrespondenz:

PD Dr. med. Dominik Heim

Chefarzt Chirurgie

Spital Frutigen

$\mathrm{CH}-3714$ Frutigen

dominik.heim[at]spitalfmi.ch
Eigentlich sind wir verwöhnt! Wenn es nach Diesel, Öl und abgestandenem Abgas riecht, und die Stahltreppe etwas glitschig vom Salzwasser ist, dann ist die Insel im frühen Frühling schon ganz nahe. Ein paar Stunden nur ist es her, dass der Zug früh am Morgen Richtung Süden losgefahren ist, er war - wie immer überfüllt, in Milano dann das Südgefühl, der Espresso in der riesigen Bahnhofhalle, und der «frecciarossa» auf Binario 9. Jetzt windet es ziemlich an der Reling, die Mammutkappe erinnert noch an die Berge, es wird Abend auf der Insel.

$\mathrm{Ob}$ Fernweh schweizerisch ist, ist sehr fraglich, Tatsache ist, dass wir mit unserer mitteleuropäischen Lage diesem Weh gut nachgeben können.

Harold hat diesem Gefühl in Rachel Joyce' «The unlikely pilgrimage of Harold Fry» (2012), wenn auch aus einem ganz andern Grund, nachgegeben. Anstatt zum Briefkasten geht er (mit dem Brief immer noch in der Tasche) von Kingsbridge nach Berwick-uponTweed zu Fuss mit seinen Segelschuhen vom einen Ende Englands ans andere Ende. Es ist nicht «utterly funny», es ist «touching», und es ist zwischendurch auch voll von «tristezza», würde Roberta sagen («sono Roberta», sagte sie, als sie um eine "carta d'identità» bat), die Besitzerin des gelben Hotels in der kleinen Stadt mit den orangen und backsteinroten Häusern auf der Insel am Meer, grad an der Hafenmole neben dem mittelalterlichen Leuchtturm, wo das grosse, weisse Schiff mit der dunkelroten Schrift am Abend anlegt. Überall im Haus hängen Bilder mit Landschaften, Aktbilder, Bilder von Schiffen und Szenen aus dem Alltag der Insel. Man entdeckt sie immer wieder neu im Salon (dort steht eine Staffelei mit einem halbfertigen Ölbild), in den Gängen und im Treppenhaus es ist das Museum einer malenden Hotelfamilie in drei Generationen mit vielen Künstlerfreunden. Man nimmt hier nie den Lift, man hat zu viel zu sehen, wenn man fast lautlos über den burgunderroten, schweren Teppich läuft.

Und man freut sich über den Zufall, dass man grad hier abgestiegen ist.

Zufälle, die auch George in «the adventures of Goodnight and Loving» von Leslie Thomas (1986, leider vergriffen) auf seiner Reise um die Welt begleiten. Mehr «funny», zum Schmunzeln, nicht immer ganz wirklich, aber phantastisch (in der ganzen Bedeutung des Wortes) und das Fernweh wieder weckend. Eigentlich sind sie «roadbooks» - ganz in Analogie zu den Roadmovies («Easy Rider» und «Thelma and Louise» kommen einem da spontan in den Sinn) - diese Bücher, die vom Laufen, vom ungeplanten Reisen, vom Ausbrechen aus der Gewohnheit erzählen. Und sie be- gleiten einem «on the road» (das Kultbuch kennt man ja) auf der Fahrt ans Meer und lassen einem an die Zufälle denken, die man auch in der Gewohnheit des Berufslebens täglich erlebt, im Operationssaal (da war doch letzthin eine incarzerierte Treitz-Hernie), in der Poliklinik (jetzt musste die Tibiaschaftfraktur des 6-jährigen Kindes doch noch im Gips gekeilt werden), in der Praxis (eine 40-jährige Patientin, die versichert, dass sonst wirklich niemand in der Familie Varizen habe). Eigentlich sind wir verwöhnt, dass wir nicht während 40 Jahren in einer Brauerei gearbeitet haben (Harold Fry) oder als Anwalt einer Zeitung (George Goodnight), dass wir dafür nach der Malleolarfraktur den Lichtenstein, die Karpaltunnelspaltung mit den feinen Instrumenten nach Strickland, die Besenreiser mit etwas Aethoxysklerol nach der Crossektomie veröden und am Schluss noch die Hämorrhoidenarterienligatur machen können. Eintönigkeit ist es also nicht, die einen ans Meer treibt. Ist es das? «Mare mare mare mi basta l'aria piena di sale e questa boccata eccezionale» singt Pippo Pollina («Appartenenza», 2014) Der Cantautore verliess nach einem Jus-Studium Sizilien und zog als Strassenmusiker durch Europa («on the road»!) und wurde in Zürich sesshaft. Mit «fra due isole» (2009) hat er zusammen mit dem Jugend-Symphonieorchester des Konservatoriums Zürich auf wundersame Weise das italienische Lied mit der klassischen Musik verschmolzen - zwei musikalische Inseln!

Auf der Insel angekommen schmunzelt man, wenn der Buschauffeur dem Autofahrer, der ihm auf seiner Seite in der engen Strasse entgegenkommt, «Dementi!» entgegenschleudert. Das ist eine ganz neue Anwendung der psychiatrischen Terminologie! Am Abend in der «d'Angelo Bar» spielt ein beleibter Sänger mit schulterlangen, schwarzen Kruselhaaren in einem schwarzen Harley-Davidson-Hemd mit seinem bärtigen mittdreissiger Gitarristen und zwei älteren Herren mit schütterem Haar im Pensionsalter am Schlagzeug und am Bass. Das ist «Italianità» pur (und nicht musikalische Mainstream-Strandromantik), ein «giro» durch all die Lieder von Francesco de Gregori, Lucio Dalla und vielen andern, und das Publikum geht mit, singt mit, ja ersetzt gar den Sänger mitten im Lied, wenn er an der Bar einen «bicchiere di vino bianco» holt - nur der «Gelato al limon» von Paolo Conte wäre da schon fast zu banal gewesen. Und über dem Türrahmen der Boutique nebenan steht in ganz einfachen Worten geschrieben «la gioia di trovare, il piacere di avere» und wenn man das bildlich und nicht kommerziell versteht, dann finden das Fernweh und unser Mediziner-Beruf eine Erklärung.

Denn eigentlich sind wir schon f(v)erwöhnt! 